\title{
Exploring the Design Space of Therapeutic Robot Companions for Children
}

\author{
Cara O'Brien \\ Trinity College Dublin \\ Dublin, Ireland \\ obriec55@tcd.ie \\ Johann Issartel \\ Dublin City University \\ Dublin, Ireland \\ johann.issartel@dcu.ie
}

\author{
Molly O’Mara \\ Trinity College Dublin \\ Dublin, Ireland \\ momara@tcd.ie \\ Conor McGinn \\ Trinity College Dublin \\ Dublin, Ireland \\ mcginnc@tcd.ie
}

\begin{abstract}
Robots that lend social and emotional support to their users have the potential to extend the quality of care that humans can provide. However, developing robotic aids to address symptoms of loneliness, anxiety and social isolation can be especially challenging due to factors that are complex and multi-faceted. Using a user-centered approach, a prototype therapeutic robot, TACO, was developed. The design of this robot was closely informed by a comprehensive needfinding process which included a detailed literature review, ethical analysis, interviews with pediatric domain experts, and a site visit to a pediatric hospital. The prototype robot was evaluated over the course of several structured play sessions, using short interviews with children as well as a modified version of the SOFIT testing procedure. Results from early-stage testing suggest that TACO was well-liked; children found playing with it engaging and frequently exhibited affective behaviors like cuddling and stroking. These findings motivate follow-on work to further advance its design and to test its effectiveness as a therapeutic tool.
\end{abstract}

\section{CCS CONCEPTS}

- Computer systems organization $\rightarrow$ Robotics; - Social and professional topics $\rightarrow$ Codes of ethics; • Human-centered computing $\rightarrow$ Interaction devices; User studies.

\section{KEYWORDS}

user-centered design, assistive robot, child-robot interaction, ethics canvas

\section{ACM Reference Format:}

Cara O’Brien, Molly O’Mara, Johann Issartel, and Conor McGinn. 2021. Exploring the Design Space of Therapeutic Robot Companions for Children. In Proceedings of the 2021 ACM/IEEE International Conference on HumanRobot Interaction (HRI '21), March 8-11, 2021, Boulder, CO, USA. ACM, New York, NY, USA, 9 pages. https://doi.org/10.1145/3434073.3444669

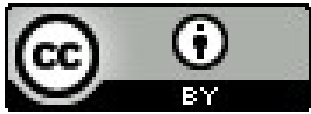

This work is licensed under a Creative Commons Attribution International 4.0 License

HRI '21, March 8-11, 2021, Boulder, CO, USA.

(C) 2021 Copyright held by the owner/author(s).

ACM ISBN 978-1-4503-8289-2/21/03.

https://doi.org/10.1145/3434073.3444669

\section{INTRODUCTION}

According to a recent report from the World Health Organisation (WHO), there is currently a global shortage of 7.2 million healthcare workers, and this number is expected to grow to 12.9 million by 2035 [48]. Addressing this problem will require new healthcare models and the development of innovative technologies that can assist with the provision of care functions.

This research was motivated by the idea that a robot might be used to assist and extend, rather than replace, human care givers in a pediatric setting. In these instances, it is proposed that robots could provide functionality that cannot be easily performed by humans, or perform during periods where humans may not be available to tend to the child. More specifically, it was envisioned that the robot may provide reassurance during prolonged hospital visits where young children (under the age of 10) frequently become socially isolated, and death and pain can be recurring daily thoughts [18], potentially affecting their mental health and development [15, 21]. It was hypothesised that when employed in the correct manner, robots might enable children to express their thoughts and emotions thereby giving them a release and helping them regain a sense of control over their life [24].

Such types of robot deliver value to their users from the experience of interacting with them, making their value harder to quantify than conventional robots developed to automate easily quantifiable practical tasks (i.e. medicine delivery, floor cleaning). Rather, the value of social care robots is based on subjective and contextual factors, depending on individual users as well as the broader use context. The ethical implications of care robots can also be significant $[16,41]$ and difficult to monitor in practise. This type of challenge is known as a 'wicked' problem [32], due to the many interdependent factors making them appear impossible to solve. Overcoming these kinds of problems requires designers to consider more than just the engineering requirements of the technology, but also the needs of the key stakeholders, the broader use context and the ethical issues that may arise from its use. Through adoption of a structured user-centered design approach, this research makes three key research contributions. First, we present findings from detailed needfinding activities into the potential suitability and applicability of a care robot in a pediatric setting. Second, using the Ethical Canvas as a tool, we identify steps to mitigate some of the most significant ethical issues that we encountered. Finally, we 
present the design and initial validation of a prototype robot for use in a pediatric setting.

\section{PRIOR WORK}

Existing research has shown that therapeutic robots (sometimes referred to by the broader term of Socially Assistive Robots (SARs)) can provide wellness benefits to a wide range of users. Due to the prevalence of dementia and other cognitive impairments among the senior population, much of the research in this area has focused on applications with older adults [2], many focusing on the use of the Paro robot [35-37, 43, 44].

Therapeutic robots have also been widely explored in the treatment of people with Autism Spectrum Disorder (ASD), where they have been shown to boost engagement and help elicit novel social behaviors [39], especially among younger people with the disorder. Kaspar is a minimally expressive social robot that was designed as a mediator for robot-assisted play and therapy for children with autism [7]. Studies with Kasper have demonstrated significant therapeutic benefits, such as facilitating collaborative games [45], encouraging interactions between children (with autism) and co-present adults [34], and high flexibility for use across many domains of ASD therapy [10]. Keepon is another interactive robot designed for children with autism. Long-term studies with Keepon revealed that its simplistic appearance encouraged spontaneous interactions with children with autism, even in unstructured environments [19]. Keepon has also been tested as a companion for children of different age groups, including infants. These studies revealed that the interactions with the robot evolved over time for different users, which if captured, might provide unique insights into the development of individual children [20].

There is a body of literature concerning robots and children in non-clinical settings. Theses studies have tended to present findings that suggest robots can be effective tools for providing emotional [22] and learning support [9, 33, 46] for children.

Although the first use of robots to help children cope with illness, invasive surgeries and hospitalisation was more than two decades ago [3], the growth in the use of therapeutic robots in a pediatric care setting has been slow. In a study by Manesh et al. which used the Nao robot, it was found that by using distraction methods such as asking questions and instructing them on breathing techniques [29], the robot was able to decrease the pain associated with vaccination injections by as much as $50 \%$ [1]. The Huggable robot, first developed in 2006, is a socially and emotionally interactive robot teddy bear that has been designed and developed to mitigate stress, anxiety and pain in pediatric patients. Experiments done using the Huggable have shown that embodied robots are perceived more positively and are more effective at persuasion than a virtual interface, and can also interact with children for longer [12]. Children were shown to interact with the Huggable by tickling, hugging, high-fiving and petting it [14]. A recent paper from the group, which involved a clinical pilot of the robot in a US pediatric hospital, indicated that social robots can be more effective at boosting mood than traditional soft toys or equivalent digital technologies [25]. In this, possibly the first long-term pilot of its type, the authors also emphasize significant practical as well as ethical challenges they encountered surrounding the use of the robot in these settings.

\section{USER-CENTERED DESIGN APPROACH}

This study was carried out using a structured user-centered design approach closely based on the ME310/SUGAR design processes; these methods were originally conceived at Stanford University [6], and have since developed into the design curriculum practised within the global 'SUGAR' ${ }^{1}$ network. Detailed needfinding was first performed to gain understanding of the user and their needs; this was followed by a range of design activities that seek to foster idea generation and design space exploration. Ideas generated in the earlier stages were then refined and developed into a tangible prototype which was then subjected to user testing and preliminary evaluation.

The project began with the formulation of a problem statement, which was then used to help structure the needfinding process. The initial problem statement was expressed as:

"How might robot technology improve the care given to children in hospital?"

\section{INSIGHTS FROM NEEDFINDING}

\subsection{Needfinding Approach}

Guided by the problem statement, a detailed needfinding process was undertaken. This process was exploratory in nature and involved investigating many of the challenges faced by children with illnesses, with a particular focus on those who are hospitalised. First a detailed literature review was undertaken. Searches were performed on ACM, IEEE, Google Scholar, Springer, Elsevier, and PubMed with a combination of the following terms: "therapeutic", "robot", "social(ly)", "assistive", "SAR", "therapy". This review informed the selection of the target primary user, children patients aged 4-8. This age group was deemed appropriate for this HRI study, as children in this age range are developmentally similar from a socio-emotional standpoint. This age stratification is also similar to those in hospital programs for children. Secondary users were staff in pediatric hospitals, while families of children in hospital, hospital management, etc. were considered tertiary users. Next, a site-visit to a national children's hospital in Ireland was conducted. During this visit, it was observed that primary and secondary users in live settings which included pediatric wards, hospital classrooms and specialized rooms for physical therapy and play therapy. Formal ethnographic methods were not used, since the objective of the exercise was to ground insights from the literature review and to familiarize ourselves with the kind of real-world environment that a pediatric therapeutic robot would be used. A semi-structured interview was conducted with a pediatric doctor and senior child life specialist. Both interviews followed a similar structure and comprised a mix of both open (i.e. "what kind of challenges do you face when bringing in new toys or play instruments?") and closed ("what is the average length of time that children stay in hospital?") questions, and were aimed primarily at deepening our understanding

${ }^{1}$ SUGAR (https://sugar-network.org) is a global network that brings together students, universities and companies to engage in human-centered innovation projects. 
of the needs/requirements of the target user. Responses to questions were noted, although the interviews were not recorded; the decision to not record these interviews was made to encourage the interviewee to speak openly without fear of reprisal. Other more informal conversations were held with children who had recently spent time in hospital and parents of children who had spent time in hospital; the subjects of these interviews were friends and family of the researchers that had used the pediatric services at some point in the past, and served to broaden our understanding of the child patient experience.

\subsection{Insights from Needfinding}

The needfinding phase provided an opportunity to get a better understanding of primary and secondary users. Some of the key insights that emerged during this period, which are of broad interest to the HRI community, include:

\section{Anxiety is very common in a pediatric setting}

It emerged from our interviews that anxiety was very common among children who spend time in hospital. These feelings can be especially difficult for children to verbalise, and are normally manifested through telling behaviours that staff learn to recognise. One of the leading causes of this anxiety is a perceived loss of control [24]. To help address this imbalance, where possible, staff interviewed in this study expressed that they make an effort to give control back to children where possible. As a simple example, different colour band-aids are always offered to the children to give them a sense of choice.

\section{Environment makes a difference}

The unfamiliar surroundings and clinical feel of hospitals can have a tangible adverse effect on the mood and anxiety experienced by child patients. Conscious efforts are often made by hospitals to make the space feel less clinical; for example, during the site visit it was observed that the walls painted with bright colors, the presence of toys in many rooms and a multi-sensory room that was designed to help children relax and escape the stress of everyday life in the hospital.

\section{Play is important, but hard in hospitals}

Throughout the needfinding process, it became clear that play is an extremely important part of everyday life for children in hospital. Play is important not only for their development, but in helping them relax and it can provide a release from the stresses of hospital life. From the site visit, it emerged that there were a range of opportunities for children to engage in play ranging from playing with their own toys in their beds, to playing with communal toys in open spaces in the hospital and also in a special multi-sensory room that contained a number of different relaxation tools such as various lighting displays (LEDs, UV light Fiber Optics), aromatherapy, interactive panels and a range of tactile objects.

Many hospitals employ child-life specialists (also referred to as play specialists), who help facilitate therapeutic play and work with children to develop coping techniques, psychological preparation, procedural support and clinical education regarding health care experiences. A meeting with the child-life specialist indicated that although all children benefited from play, the majority of bookings for the multi-sensory room were children in the 4-8 age category.
Despite the importance of play, there are many challenges that can limit the amount of time children can engage in it. Given space constraints and safety concerns, it can also be challenging for children to engage in physical or dynamic play, which has been shown to have important health benefits for children, even those with illness [31]. Dedicated play spaces like the multi-sensory room seem to offer a solution to this problem, however (in our experience) they required pre-booking and the duration children could spend there was limited to under an hour at a time. The room was in high demand and not all children that wanted to use the room could get access to it; most of the children booked into the room were ones who were extremely nervous about upcoming procedures. Also, for hygiene reasons, the type of toys that could be used in communal parts of the hospital were closely controlled; only toys that could be easily wiped were permitted.

\section{Resources are limited}

From our interviews, it was clear that staff felt under-resourced to meet the needs of all the patients. As mentioned previously, important facilities like the multi-sensory room have limited availability, which can severely limit the number of opportunities for children to get respite from their hospital beds. Additionally, chronic understaffing can often lead to children spending much of the day by themselves, without the company and social support of another person. Furthermore, when the facility is understaffed, it can be difficult for staff to pick up on behavioural changes or non-verbal triggers that might indicate that a child needs extra attention and support.

Robotic interventions still in early stages

As detailed in section 2, therapeutic robots have shown potential to reduce stress and anxiety in children, especially in the case where they are integrated with existing therapeutic activities. However, there are still numerous limitations that must be overcome before the full value of these robots can be realised.

Until very recently, therapeutic robots deployed in field studies have been significantly constrained by their weight, size and lack of mobility [13, 38, 40]. Only in recent months has the Huggable, arguably the best known pediatric robot, become portable; previous versions were immobile and tethered to a host computer ensuring that it could not be picked up and carried by child users. Despite the portability of the newer model, to the best of the authors knowledge, Huggable has not been involved in any trials that involve long-term sustained physical interactions with users.

It is also common in the literature for therapeutic robots to lack autonomy $[4,8,11,17,25,34]$ and for tests to be conducted in highly controlled settings that are not likely to provoke the kinds of interactions that would be observed in the real world $[13,40]$.

Another problem observed in the literature is the lack of standardised age appropriate procedures and metrics for testing robots with children. This is especially important considering that young, sick children represent a highly heterogeneous participant population and necessitates detailed consideration of the testing procedures used.

\subsection{Understanding Ethical Landscape}

The Ethics Canvas (EC) is a collaborative brainstorming tool with the overall aim to foster ethically informed technology design by 


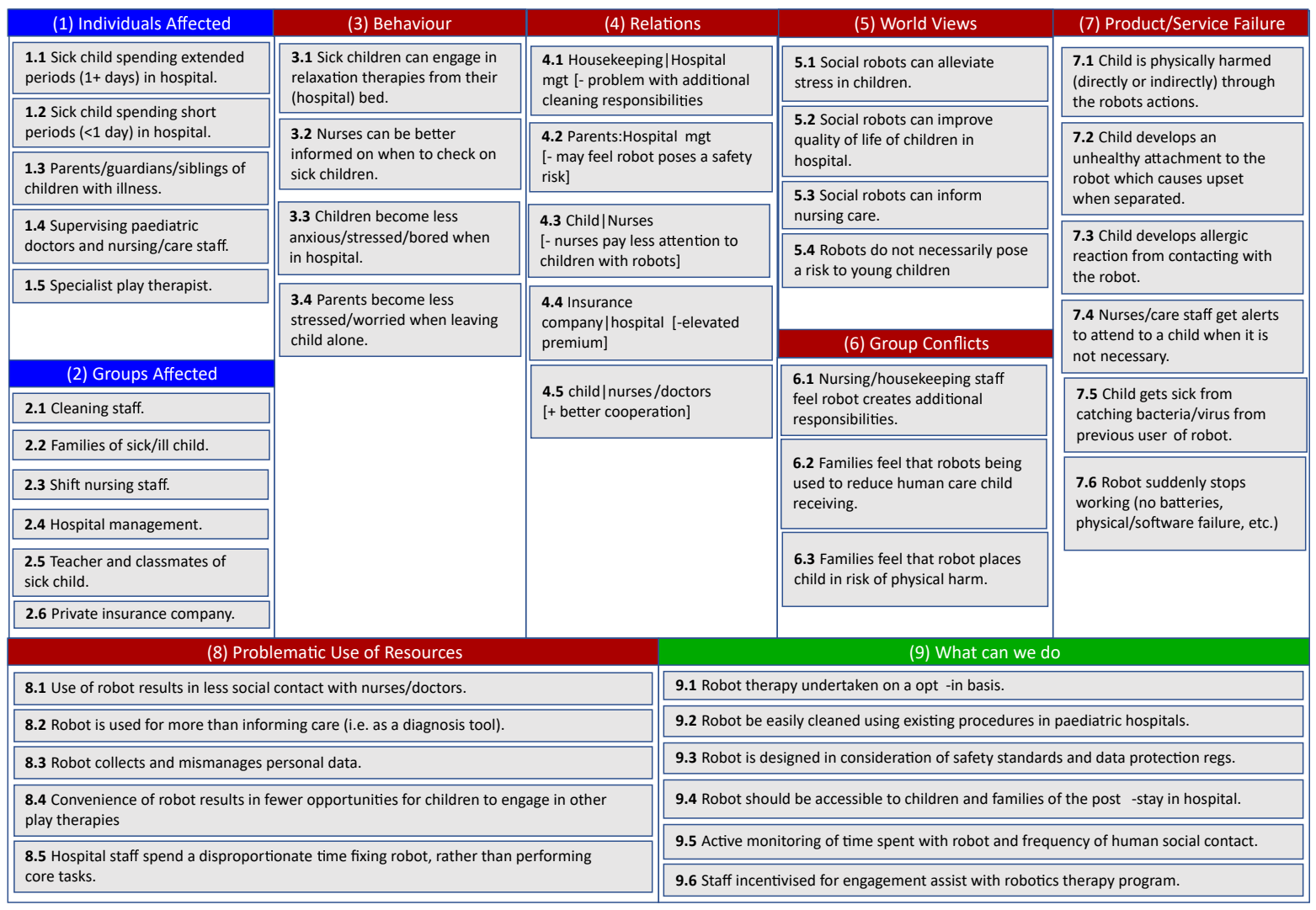

Figure 1: Completed Ethics Canvas for pediatric robot use-case.

improving the engagement of research and innovation practitioners with the ethical impacts of their activities [30]. The development of the EC was inspired by a widely used business-modelling method known as the Business Model Canvas (BMC) [28], a visual-linguistic tool that captures key elements of a business model into nine thematic building blocks on a one page document. To the best of our knowledge, it has yet to be applied in a robotics context.

The EC has nine 'building blocks' and is orientated to help answer three basic questions: (1) Who might be affected by the technology? [blocks 1,2], (2) What are the potential ethical impacts for these people and groups [blocks 3-8], and (3) how can we address these ethical impacts? [block 9] [23].

The EC for this use-case was compiled and refined over a period of several months. In most instances, data compiled in the EC is grounded in insights gained from speaking with physicians and people who shared experiences from use of pediatric services. Updates to the EC were made over the course of the project and emerged from on-going reflection and gaining new insights and improved overall understanding of the use context. In this section, key ethical recommendations (block 9), which address potential impacts (blocks 3-8), are identified and briefly discussed. A copy of the most recent Ethics Canvas prepared for this study is given in figure 1 .
The following recommendations are made to support ethical long-term deployment of the technology:

- Access to the robot should be provided on an opt-in basis, with consent provided from guardians and an initial trial period to monitor the child's acceptance of the technology. [Main Issues addressed: 3.4, 4.2, 6.2, 6.3]

- The robot should be cleanable using the existing hygiene practices in pediatric hospitals. [Main Issues addressed: 4.4, $6.1,7.3,7.5,8.5]$

- The robot should be developed in consideration of appropriate data protection and medical device and/or product safety standards. [Main Issues addressed: 4.2, 4.4, 5.4, 6.3, 7.1, 7.3, 7.6, 8.3, 8.5]

- Versions of the robot should be made accessible to children and families after leaving the hospital (if required). [Main Issues addressed: $3.1,3.4,7.2$ ]

- Monitoring procedures should be put in place to ensure that children that use the robot do not receive reduced levels of human contact or develop unhealthy bonds with robot. [Main Issues addressed: 3.3, 3.4, 4.2, 4.3, 4.4, 6.2, 6.3, 7.2, 8.1]

- Incentives (professional/financial) are provided to staff to engage with the robotics program. [Main Issues addressed: $4.1,4.3,4.5,6.1,7.4,8.1]$ 


\section{PROTOTYPE DESIGN}

\subsection{Ideation and Design Space Exploration}

Over a period of several months, an overall design concept was formulated. The robot was named TACO, which is an acronym for Therapeutic Active COmpanion. This concept evolved through many cycles of idea generation, facilitated by activities such as brainstorming, sketching and low resolution prototyping. Some examples of early design concepts included:

- A robot ball that could be kick around to vent frustration, but then soft enough to cuddle.

- A soft robotic toy with naturally compliant actuation.

- A modular toy robot that children could cuddle, but could also be reconfigured to engage the user in physical activity by moving around on the floor.

A table summarising the most important user-needs that emerged from our needfinding analysis, the existing state of the art that best addresses them, and how the features were subsequently implemented on the design of TACO is given in table 1 . It is observed that only TACO meets each of the identified requirements.

\subsection{Critical Function and Critical Experience Prototyping}

Exploring these concepts led to the development of two distinct types of prototype; the critical function prototype (CFP) and critical experience prototype (CEP). The purpose of a CFP is to explore key functional requirements of the overall system, while the CEP allows for investigation of the most important experiential element. In this project, the CFP initially investigated approaches for making a therapeutic robot 'soft' and compliant, which was deemed important for tactile interactions. A pneumatic solution was first explored, whereby an air pump, housed inside the robot, inflated bellows which acted as a cushion. A low resolution prototype was created to test this idea, and although this approach was ultimately dropped due to several practical limitations (i.e. need for additional hardware, low efficiency of pump), it generated a valuable insight; the cyclic expansion and contraction of the system, produced by manipulating the inlet and outlet valves, had an engaging and surprisingly reassuring effect on most users that held it. This provided some early evidence that incorporating a cyclic movement, which resembled a breathing action, might have positive effect on human-robot interaction. This prediction is supported by prior research that the experience of sensing someone breathing can have a calming and comforting effect $[42,49]$. Additionally, a portable 'breathing' robot is physically well suited for a hospital environment, and it poses relatively less risk than other actuated morphologies that might roll or move around. Consequently, the CFP was pivoted to investigate the best way to incorporate a cyclic 'breathing-like' action. It was subsequently found that a slider-crank mechanism proved to be both simple to make and possessed the required mechanical properties.

The objective of the CEP was to explore what features the robot should possess to provide a feeling of calm and comfort to the users when they interact with it. This CEP was inspired by research which indicates physical warmth and touch can have comforting effects on people $[5,20,40,47]$. The CEP investigated if a robot could provide a reassuring sensation through physical contact with a user. To explore this on a robot, a low resolution prototype was developed which involved integrating electrically controlled heat pads inside a foam pillow. Observation and verbal feedback given during informal testing of the prototype suggested that people generally enjoyed the sensation produced; commonly observed behaviours were hugging and placing their hands over the area that was heated. This testing was mostly done with university students or researchers in the department that the project was conducted, so while the tests did not provide scientifically rigorous validation, it provided a fast route to better understand the hedonic aspects of the design feature.

\subsection{Design of TACO}

The final design concept involved a non-anthropomorphic robot that was small $(47 \times 26 \times 16 \mathrm{~cm})$ and light enough for a child to carry $(<3 \mathrm{~kg})$. This size was determined to be large enough that it provided a sense of presence, but small enough to be portable and non-intimidating. To avoid a design that may deceive the child, or encourage an unhealthy attachment to form between the child and the robot, a conscious decision was made to make the robot in such a way that there would be no obvious visual association between it and a human or animal. To provide the robot with a 'soft' feel, the structure was encased in a layer of cushioned memory foam. Finally, the robot was covered in a removable soft textile that was custom tailored to fit the contours of the robot. To mitigate biasing effects, a neutral white-gray-black color scheme was chosen for the outer layer of the robot. The robot was designed to exhibit a range of reassuring behaviours which could be triggered autonomously when the robot was being held, as determined by changes in the reading of an on-board IMU. These behaviours included a heating effect and breathing mechanism (informed by findings described in section 5.2), as well as a RGB lighting. The latter feature was included to enhance the perception of animacy of the robot, and it was also hypothesised that lighting might have a positive influence [26, 29] on mood. Figures 2(a) - 2(c) illustrate how the breathing, lighting and heating effects were implemented.

\section{EVALUATION}

\subsection{Experimental Design}

An exploratory study was undertaken with TACO. As this was the first time the robot was tested with children resembling the target demographic, the decision was made not to test the robot in a hospital or around potentially vulnerable users. Instead, tests were performed in a classroom at a children's elementary school. This setting avoided some of the significant risks and challenges of testing at a hospital, while providing a familiar public space normally populated by children. As a result, the goal of this initial study was not to validate the effectiveness of the robot as a therapeutic aid, but to explore the first impressions that children formed of the robot, and to better understand the suitability of the robot's ergonomics. This latter question was especially interesting given the gap of literature on physical human-robot interaction with children. Ethical approval to conduct these tests was attained by the university research ethics committee, and written informed 
Table 1: Identification of key user requirements and how they are met by TACO's design.

\begin{tabular}{|c|c|c|c|}
\hline User Need & Motivation & Prior Art & Implementation on TACO \\
\hline Portable & $\begin{array}{l}\text { Large body of research indicates practical and thera- } \\
\text { peutic benefits of robots being easily carried. }\end{array}$ & $\begin{array}{l}\text { Keepon, Paro, } \\
\text { Nao, Huggable }\end{array}$ & $\begin{array}{l}\text { Designed to be carried by children and easily } \\
\text { placed on lap (dimensions } 47 \times 26 \times 16 \mathrm{~cm} \text { ). }\end{array}$ \\
\hline $\begin{array}{l}\text { Provide Reassur- } \\
\text { ance and Comfort }\end{array}$ & $\begin{array}{l}\text { Stress, anxiety and feelings of isolation in children } \\
\text { may be reduced if the robot provides a comforting } \\
\text { presence. }\end{array}$ & $\begin{array}{l}\text { Haptic Creature, } \\
\text { Huggable, Probo, } \\
\text { Paro }\end{array}$ & $\begin{array}{l}\text { Soft, cushioned feel. Produces warm sensation } \\
\text { through heat pads. Produces a cyclic movement, } \\
\text { similar to breathing. }\end{array}$ \\
\hline Easily Cleaned & $\begin{array}{l}\text { Must be capable of being disinfected on a regular } \\
\text { basis. }\end{array}$ & $\begin{array}{l}\text { Huggable, Probo, } \\
\text { Paro }\end{array}$ & $\begin{array}{l}\text { The case of TACO is easily replaced, and can be } \\
\text { machine washable. }\end{array}$ \\
\hline Easy-to-Use & $\begin{array}{l}\text { Designed for } 4-8 \text {-year old children so must be easy } \\
\text { for them operate. }\end{array}$ & Keepon, Paro & $\begin{array}{l}\text { Simple repetitive behavior, initiated once the ro- } \\
\text { bot is picked up. }\end{array}$ \\
\hline $\begin{array}{l}\text { Sensory Stimula- } \\
\text { tion }\end{array}$ & $\begin{array}{l}\text { Tactile and visual stimulation can be comforting to } \\
\text { children and provide therapeutic value. }\end{array}$ & $\begin{array}{l}\text { Huggable, Haptic } \\
\text { Creature, Probo }\end{array}$ & $\begin{array}{l}\text { Designed to provide tactile experiences through } \\
\text { holding the robot and visual experiences through } \\
\text { its color scheme and LED illumination sequences. }\end{array}$ \\
\hline Long-term use & $\begin{array}{l}\text { Must be able to provide comfort for hours at a time, } \\
\text { filling gaps in the day where children are alone. }\end{array}$ & $\begin{array}{l}\text { Keepon, Paro, } \\
\text { Nao }\end{array}$ & $\begin{array}{l}\text { Robust design. Battery life is nominally } 4 \text { hours } \\
\text { but can upgraded to last longer if needed. }\end{array}$ \\
\hline Autonomy & $\begin{array}{l}\text { The robot must be able to interact without the need } \\
\text { of teleoperation, since it is to be used when there are } \\
\text { no adults there to comfort the child. }\end{array}$ & Keepon, Paro & $\begin{array}{l}\text { Fully autonomous operation, implemented } \\
\text { within a simple reactive control system. }\end{array}$ \\
\hline Customizable & $\begin{array}{l}\text { Children in hospital tend to lack a sense of control. } \\
\text { The children should be able to control the robots ap- } \\
\text { pearance and how they choose to interact with the } \\
\text { robot. }\end{array}$ & Paro, Pebbles & $\begin{array}{l}\text { The outer case for TACO can be made in differ- } \\
\text { ent color schemes and patterns, and potentially } \\
\text { designed in a bespoke way for each child that has } \\
\text { one. }\end{array}$ \\
\hline
\end{tabular}

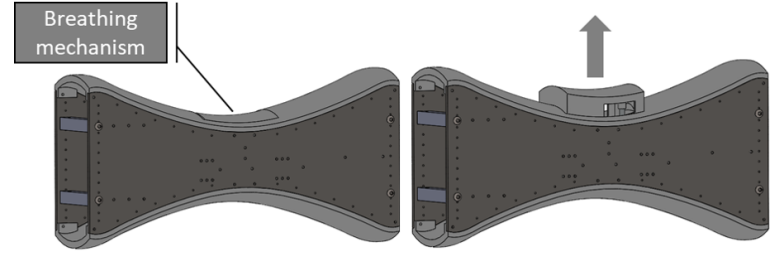

(a)

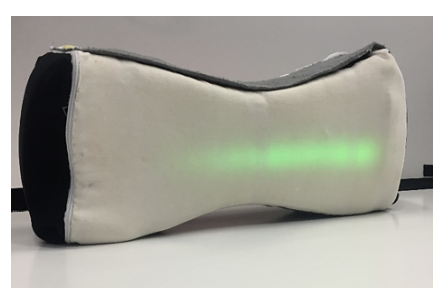

(b)

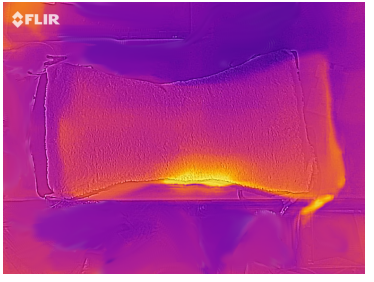

(c)
Figure 2: The design of TACO: (a) Illustration of how the 'breathing' effect was achieved, (b) photo of TACO with led lighting effect, (c) thermal image of TACO indicating heating effect.

consent was provided by the parents of the participants prior to each session.

All tests took place in the same room. The experiment was introduced to the participants as research to develop new fun toys. Participants were informed that there would be a number of activities for them to do and that they had only a short amount of time to spend at each activity. They were told that there was no right or wrong way to do any of them, and there was no good or bad result. Chairs were placed behind each of the stations so that the children could sit if they wished to. Participants were not told what to do at each station, but if they asked, some encouragement was given. This introduction was given by one of the school teachers in order to bring as little attention as possible to the observers. Any further questions from participants were held until the debriefing at the end of the session.

There were five stations set up, each with a different activity; colouring pictures, a word search, assembling Lego, playing with a magnetic construction toy, and interacting with the TACO robot prototype. Five or fewer children were admitted to each round of testing. Children spent two minutes at each station and were then moved onto the next one. After every child in the group had visited each station, a short focus group took place. It was then explained to the participants that we were especially interested in their experience of interacting with TACO, a new robotic companion we were developing for children in hospital. During the debrief, we asked each child some open questions, namely what they liked or disliked about the robot, if there was anything they wished the robot could do, and offered to answer any questions that the children may have had. This was done to see what their initial reactions were, and to gain some insights into the suitability of the robot's design for children.

During the main part of the experiment, only the station with TACO was observed; the other stations were included to distract focus from the robot, as children may have reacted differently if they knew we were only interested in the robot. A modified version of the System for Observing Fitness Instruction Time (SOFIT) [27] method 
was used; this is a widely used, validated scale for measuring child activity. It was not deemed necessary to video record the sessions.

Two researchers (both had recent prior experience performing the SOFIT procedure) sat at a distance facing all stations, not just the station with the robot on it. The observation design consisted of alternately 'observing' and 'recording' in intervals of 5 seconds; this resulted in 6 observations per minute. Data recorded during the observation was: gender, student activity and interactive activity. The student activity was the level or body position of the target participant. This was coded into one of five categories (lying, sitting, standing, walking/moderate physical activity, and vigorous physical activity).

The interactive activity of a participant was coded into one of the following six categories:

- Stroking (S): Children repeatedly pet the robot's body.

- Poking (P): Children poke the robot's body.

- Cuddling (C): Children hug the robot, place their head on it, squeeze it.

- Holding (H): Children simply hold the robot in both arms.

- Talking (T): Children speak to the robot directly.

- One Hand (O): Children hold the robot but with one arm only.

\subsection{Results}

In total, 16 children (male, female) aged between 6-9 participated in testing. It is noted that observational notes from one child were excluded from analysis due to an unscheduled interruption that took place.

All features were active during the testing so the full effect of the robot's presence could be evaluated and helped ensure equal test conditions were given to each participant. Table 3(b) summarizes how all 15 participants interacted with TACO. Holding the robot with two arms was the most common interaction type (53.3\%), followed by holding it with one arm (15.5\%), cuddling (12.2\%) and stroking it (11.6\%). Talking to the robot was the least recorded interaction type $(1.1 \%)$.

During most interactions, children were either sitting (52\%) or standing (47\%), and very occasionally walking or engaging in moderate activity (1\%). Table 3(b) summarizes the body positions of children during their interactions with TACO. In figure 3(c), the percentage of time that each participant was observed to be interacting with the robot is shown. Note that the maximum number of observations that could be recorded in the two minutes was 12 . Of the 15 participants, 13 of them interacted with the robot $100 \%$ of the time. The lowest participation recorded was that of participant 11 who interacted for only $75 \%$ of the time.

\subsection{Insights from Focus Groups with Children}

Most of the children, when asked, said there was nothing they did not like about the experience of interacting with TACO. However, when asked about features they would like on the robot, several children suggested brighter colors and indicated that they would have preferred if TACO's appearance was more anthropomorphic.

When asked what they liked most about the robot, feedback indicated that most children liked the heating pad effect (10 children), breathing movement ( 9 mentions) and the LED lighting (8 mentions). A significant portion of the test group also expressed that they enjoyed the soft tactile sensation of the robot (5 mentions). Despite the fact that the breathing mechanism made an audible noise (due to interaction of motor and transmission elements), the sounds the robot made were not referenced by any of the participants in the sample.

\section{DISCUSSION}

The results from the user testing experiment indicated high levels of engagement with TACO. Children were observed to be active for the entirety of the time, with standing and sitting being the two most popular activities. Over $85 \%$ of the participants were engaged in interaction for the full two minutes. There was some variance in the interaction activity over time, however, the most common activity was holding the robot with two hands, similar to the way illustrated in Figure 4. Children also commonly held the robot with one hand, stroked it and cuddled it throughout the interaction, however very few were observed to talk to or poke the robot. When asked about the robot, none of the participants had any negative comments, and the majority complimented the feeling of warmth provided by the heat pads, the breathing movement, and the LED lighting effect.

The experiment revealed that TACO created pleasant, engaging interactions with children, however the tests were not sufficient to show a therapeutic effect. As such, we cannot yet claim that that TACO can positively affect the mood or wellbeing of childen. However, these early findings provide support for the hypothesis that TACO may have therapudic value, and they motivate follow-on work which will involve longer term deployments of the technology.

Another significant limitation of the study was the fact that the robot was not tested with actual target users (i.e. children in hospital) or in a target usage setting (i.e. a hospital). As such, while the results indicate that TACO was well liked and children found it engaging, it is possible that different results would be observed in clinical settings where the testing population may be experiencing the effects of illness as well as significantly higher levels of anxiety. However, testing robots with young children with illness brings many challenges, and supported by the recommendations suggested in the ethical canvas, we do not believe that it would have been ethically appropriate for the first tests to be conducted in a clinical setting or around vulnerable users.

During the testing, several technical limitations in TACO's design were observed. These must be overcome in order for TACO to be safely and reliably tested in clinical settings. Firstly, the outer surface on TACO is made from textile materials which must be machine washed. This material would not be permitted in a pediatric hospital, since existing cleaning protocols only allow for items that can be easily wiped down. Future versions of the robot should use a wipeable antimicrobial material. Other design revisions to improve safety include a more secure housing to limit access to internal components, an externally accessible charging port (or inductive charging capability) and low-battery warning notification.

Related to ergonomics and usability, the motors and transmission unit actuating the breathing mechanism were quite noisy; a redesigned version of the platform should take care to ensure a 


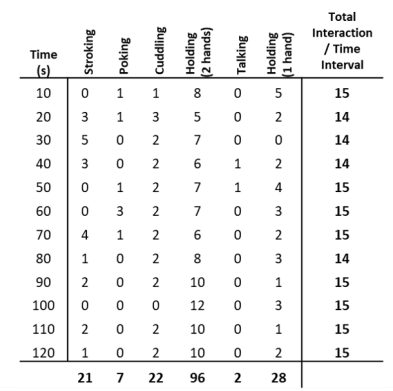

(a)

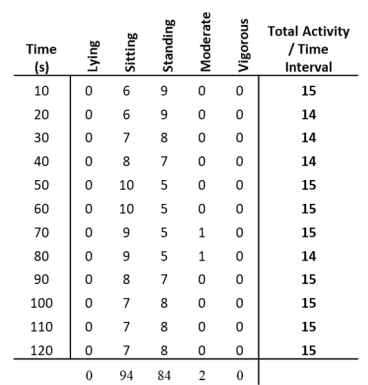

(b)

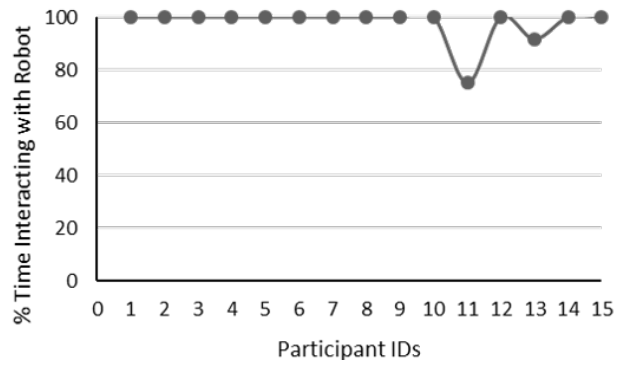

(c)

Figure 3: Results from interactions with TACO (a) recorded interactive activities from SOFIT procedures, (b) recorded student activities from SOFIT procedures, (c) recorded engagement with the robot of over duration of play slot.

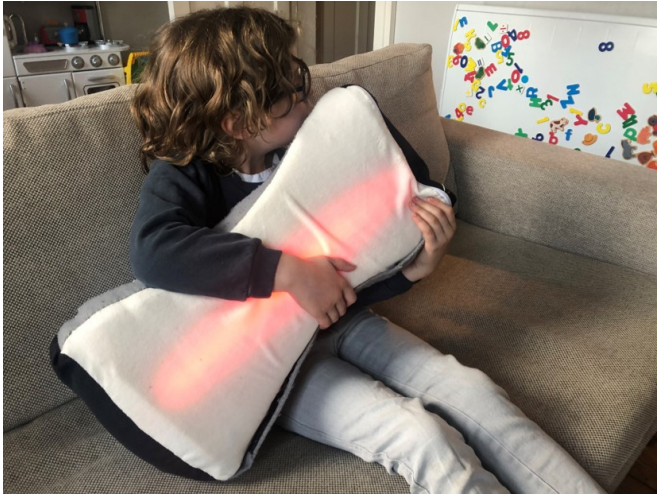

Figure 4: Photo of typical interaction with child and TACO.

quieter system. A future version of TACO should try to reduce its mass to make it easier for children to carry. TACO's appearance also needs to be re-assessed. The design was purposefully void of any visual human or animal features, however, testing suggested that children may have preferred if TACO's appearance was more anthropomorphic.

\section{CONCLUSIONS}

Despite many recent advancements in the field of robotics, there are currently very few robots that can cater specifically to the needs of hospitalized or chronically ill children. Through needfinding and benchmarking, the benefits of robot-assisted therapy were explored and a specific user was identified: 4 to 8-year-old children in hospital. In particular, it was noted that current therapeutic robots are lacking in several areas that are key to the user's requirements, these areas include: portability, physical interaction, autonomy, and sensory stimulation. From this exploration, numerous design concepts were investigated and tested to identify the most effective ways to meet the user's needs. The design concept was proposed that met these requirements, and a prototype was developed. During user-testing, it was found that children were generally engaged by the device, which was indicated by the fact that over $80 \%$ of participants remained actively interacting with it for the entirety of the testing time. Children generally showed affection towards the robot, engaging in activities such as petting and stroking, and commending the physical warmth of the device. This provides support to the idea that mechanically simple, non-anthropomorphic robots can provide comfort and reassurance to young children. These findings highlight an exciting area of research and one that warrants continued investigation.

\section{REFERENCES}

[1] Setareh Aghel Manesh, Tanya Beran, Ehud Sharlin, and Saul Greenberg. 2014. Medi, human robot interaction in pediatric health. In CHI'14 Extended Abstracts on Human Factors in Computing Systems. ACM, 153-154.

[2] Roger Bemelmans, Gert Jan Gelderblom, Pieter Jonker, and Luc De Witte. 2012. Socially assistive robots in elderly care: A systematic review into effects and effectiveness. Fournal of the American Medical Directors Association 13, 2 (2012), 114-120.

[3] Marina Umaschi Bers, Edith Ackermann, Justine Cassell, Beth Donegan, Joseph Gonzalez-Heydrich, David Ray DeMaso, Carol Strohecker, Sarah Lualdi, Dennis Bromley, and Judith Karlin. 1998. Interactive storytelling environments: coping with cardiac illness at Boston's Children's Hospital. In Proceedings of the SIGCHI Conference on Human Factors in Computing Systems. ACM Press/Addison-Wesley Publishing Co., 603-610.

[4] John Travis Butler and Arvin Agah. 2001. Psychological effects of behavior patterns of a mobile personal robot. Autonomous Robots 10, 2 (2001), 185-202.

[5] John-John Cabibihan, Rangarajan Jegadeesan, Saba Salehi, and Shuzhi Sam Ge. 2010. Synthetic skins with humanlike warmth. In International Conference on Social Robotics. Springer, 362-371.

[6] T Carleton and L Leifer. 2009. Stanford's ME310 course as an evolution of engineering design. In Proceedings of the 19th CIRP Design Conference-Competitive Design. Cranfield University Press.

[7] Kerstin Dautenhahn, Chrystopher L Nehaniv, Michael L Walters, Ben Robins, Hatice Kose-Bagci, N Assif Mirza, and Mike Blow. 2009. KASPAR-a minimally expressive humanoid robot for human-robot interaction research. Applied Bionics and Biomechanics 6, 3-4 (2009), 369-397.

[8] Marta Díaz, Neus Nuno, Joan Saez-Pons, Diego E Pardo, and Cecilio Angulo. 2011. Building up child-robot relationship for therapeutic purposes: From initial attraction towards long-term social engagement. In Face and Gesture 2011. IEEE, 927-932.

[9] Goren Gordon, Samuel Spaulding, Jacqueline Kory Westlund, Jin Joo Lee, Luke Plummer, Marayna Martinez, Madhurima Das, and Cynthia Breazeal. 2016. Affective Personalization of a Social Robot Tutor for Children's Second Language Skills.. In AAAI. 3951-3957.

[10] Claire AGJ Huijnen, Monique AS Lexis, and Luc P de Witte. 2016. Matching robot KASPAR to autism spectrum disorder (ASD) therapy and educational goals. International fournal of Social Robotics 8, 4 (2016), 445-455.

[11] Sooyeon Jeong. 2014. Developing a social robotic companion for stress and anxiety mitigation in pediatric hospitals. Ph.D. Dissertation. Massachusetts Institute of Technology.

[12] Sooyeon Jeong. 2017. The impact of social robots on young patients' socio-emotional wellbeing in a pediatric inpatient care context. Ph.D. Dissertation. Massachusetts Institute of Technology. 
[13] Sooyeon Jeong, Deirdre Logan, Matthew Goodwin, Suzanne Graca, Brianna O'Connell, Laurel Anderson, Honey Goodenough, Nicole Stenquist, Alex A Ahmed, Duncan Smith-Freedman, et al. 2015. Challenges conducting child-robot interaction research in a pediatric inpatient care context. In The First Workshop on Evaluating Child-Robot Interaction Held in Conjunction with the Seventh International Conference on Social Robotics.

[14] Sooyeon Jeong, Deirdre E Logan, Matthew S Goodwin, Suzanne Graca, Brianna O'Connell, Honey Goodenough, Laurel Anderson, Nicole Stenquist, Katie Fitzpatrick, Miriam Zisook, et al. 2015. A social robot to mitigate stress, anxiety, and pain in hospital pediatric care. In Proceedings of the Tenth Annual ACM/IEEE International Conference on Human-Robot Interaction Extended Abstracts. ACM, 103-104.

[15] Zeev N Kain, Linda C Mayes, Alison A Caldwell-Andrews, David E Karas, and Brenda C McClain. 2006. Preoperative anxiety, postoperative pain, and behavioral recovery in young children undergoing surgery. Pediatrics 118, 2 (2006), 651-658.

[16] Kenneth Kernaghan. 2015. Serving seniors: Innovation and public sector service delivery. The Innovation fournal 20, 2 (2015), 2.

[17] Elizabeth S Kim, Lauren D Berkovits, Emily P Bernier, Dan Leyzberg, Frederick Shic, Rhea Paul, and Brian Scassellati. 2013. Social robots as embedded reinforcers of social behavior in children with autism. Fournal of autism and developmental disorders 43, 5 (2013), 1038-1049.

[18] Konstantinos Koukourikos, Laila Tzeha, Parthenopi Pantelidou, and Areti Tsaloglidou. 2015. The importance of play during hospitalization of children. Materia socio-medica 27, 6 (2015), 438.

[19] Hideki Kozima, Marek P Michalowski, and Cocoro Nakagawa. 2009. Keepon: A playful robot for research, therapy, and entertainment. International fournal of Social Robotics 1, 1 (2009), 3-18.

[20] Hideki Kozima, Cocoro Nakagawa, and Yuriko Yasuda. 2005. Interactive robots for communication-care: A case-study in autism therapy. In Robot and human interactive communication, 2005. ROMAN 2005. IEEE International Workshop on IEEE, 341-346.

[21] Rebecca E Lacey, Meena Kumari, and Mel Bartley. 2014. Social isolation in childhood and adult inflammation: evidence from the National Child Development Study. Psychoneuroendocrinology 50 (2014), 85-94.

[22] Iolanda Leite, Ginevra Castellano, André Pereira, Carlos Martinho, and Ana Paiva 2014. Empathic robots for long-term interaction. International fournal of Social Robotics 6, 3 (2014), 329-341.

[23] David Lewis, Wessel Reijers, Harshvardhan Pandit, and Wessel Reijers. 2017. Ethics Canvas Manual.

[24] Leslie S Liu, Kori M Inkpen, and Wanda Pratt. 2015. I'm not like my friends: understanding how children with a chronic illness use technology to maintain normalcy. In Proceedings of the 18th ACM Conference on Computer Supported Cooperative Work \& Social Computing. ACM, 1527-1539.

[25] Deirdre E Logan, Cynthia Breazeal, Matthew S Goodwin, Sooyeon Jeong, Brianna O'Connell, Duncan Smith-Freedman, James Heathers, and Peter Weinstock. 2019. Social Robots for Hospitalized Children. Pediatrics (2019), e20181511.

[26] CLB McCloughan, PA Aspinall, and RS Webb. 1999. The impact of lighting on mood. International fournal of Lighting Research and Technology 31, 3 (1999), $81-88$.

[27] Thomas L McKenzie, James F Sallis, and Philip R Nader. 1992. SOFIT: System for observing fitness instruction time. Fournal of teaching in physical Education 11, 2 (1992), 195-205

[28] Alexander Osterwalder and Yves Pigneur. 2010. Business model generation: a handbook for visionaries, game changers, and challengers. John Wiley \& Sons.

[29] Daniel J Rea, James E Young, and Pourang Irani. 2012. The Roomba mood ring: an ambient-display robot. In Proceedings of the seventh annual ACM/IEEE international conference on Human-Robot Interaction. ACM, 217-218.

[30] Wessel Reijers, Kevin Koidl, David Lewis, Harshvardhan J Pandit, and Bert Gordijn. 2018. Discussing ethical impacts in research and innovation: The ethics canvas. In IFIP International Conference on Human Choice and Computers. Springer, 299-313.
[31] William F Riner and Sarah Hunt Sellhorst. 2013. Physical activity and exercise in children with chronic health conditions. Fournal of sport and health science 2,1 (2013), 12-20.

[32] Horst WJ Rittel and Melvin M Webber. 1973. Dilemmas in a general theory of planning. Policy sciences 4, 2 (1973), 155-169.

[33] David Robert and Cynthia Breazeal. 2012. Blended reality characters. In Proceedings of the seventh annual ACM/IEEE international conference on Human-Robot Interaction. ACM, 359-366.

[34] Ben Robins, Kerstin Dautenhahn, and Paul Dickerson. 2009. From isolation to communication: a case study evaluation of robot assisted play for children with autism with a minimally expressive humanoid robot. In Advances in ComputerHuman Interactions, 2009. ACHI'09. Second International Conferences on. IEEE, 205-211.

[35] Hayley Robinson, Bruce MacDonald, Ngaire Kerse, and Elizabeth Broadbent. 2013. The psychosocial effects of a companion robot: a randomized controlled trial. Fournal of the American Medical Directors Association 14, 9 (2013), 661-667.

[36] Selma Sabanovic, Casey C Bennett, Wan-Ling Chang, and Lesa Huber. 2013. PARO robot affects diverse interaction modalities in group sensory therapy for older adults with dementia. In Rehabilitation Robotics (ICORR), 2013 IEEE International Conference on. IEEE, 1-6.

[37] T Saito. 2002. Examination of Change of Stress Reaction by Urinary Tests of Elderly before and after Introduction of Mental Commit Robot to an Elderly Institute. In Proc. of the $7^{\wedge}<$ th $>$ Int'l Symp. on Artificial Life and Robotics, 2002, Vol. 1. 316-319.

[38] Jelle Saldien, Kristof Goris, Selma Yilmazyildiz, Werner Verhelst, and Dirk Lefeber. 2008. On the design of the huggable robot Probo. Fournal of Physical Agents Vol. 2, No. 2 (2008), 3-11.

[39] Brian Scassellati, Henny Admoni, and Maja Matarić. 2012. Robots for use in autism research. Annual review of biomedical engineering 14 (2012), 275-294.

[40] Yasaman Sefidgar, Karon MacLean, Steve Yohanan, HF Machiel Van der Loos, Elizabeth Croft, et al. 2016. Design and evaluation of a touch-centered calming interaction with a social robot. IEEE Transactions on Affective Computing 1 (2016), $1-1$.

[41] Amanda Sharkey. 2014. Robots and human dignity: a consideration of the effects of robot care on the dignity of older people. Ethics and Information Technology 16, 1 (2014), 63-75.

[42] Evelyn B Thoman, Kelsy Hammond, Glenn Affleck, and Hema N Desilva. 1995. The breathing bear with preterm infants: effects on sleep, respiration, and affect. Infant mental health journal 16, 3 (1995), 160-168.

[43] Kazuyoshi Wada, Takanori Shibata, Toshimitsu Musha, and Shin Kimura. 2008. Robot therapy for elders affected by dementia. IEEE Engineering in medicine and biology magazine 27, 4 (2008).

[44] Kazuyoshi Wada, Takanori Shibata, Tomoko Saito, Kayoko Sakamoto, and Kazuo Tanie. 2005. Psychological and social effects of one year robot assisted activity on elderly people at a health service facility for the aged. In Robotics and Automation, 2005. ICRA 2005. Proceedings of the 2005 IEEE International Conference on. IEEE, 2785-2790.

[45] Joshua Wainer, Ben Robins, Farshid Amirabdollahian, and Kerstin Dautenhahn. 2014. Using the humanoid robot KASPAR to autonomously play triadic games and facilitate collaborative play among children with autism. IEEE Transactions on Autonomous Mental Development 6, 3 (2014), 183-199.

[46] Jacqueline Kory Westlund, Leah Dickens, Sooyeon Jeong, Paul Harris, David DeSteno, and Cynthia Breazeal. 2015. A comparison of children learning new words from robots, tablets, \& people. In Conference Proceedings New Friends 2015.

[47] Christian JAM Willemse. 2015. A warm touch of affect?. In Affective Computing and Intelligent Interaction (ACII), 2015 International Conference on. IEEE, 766-771.

[48] World Health Organisation. 2013. A Universal Truth: No Health Without a Workforce. (2013).

[49] Steve Yohanan and Karon E MacLean. 2009. A tool to study affective touch. In CHI'09 Extended Abstracts on Human Factors in Computing Systems. ACM, 4153-4158. 